\title{
reviscafuences
}

ISSN: 1575-7072 | e-ISSN: 2172-7775

Páginas: $13-23$

Recibido: 2019-03-12

Revisado: 2019-11-06

Aceptado: 2019-11-24

Preprint: 2020-04-20

Publicación Final: 2020-06-15

DOI: https://doi.org/10.12795/revistafuentes.2020.v22.i1.02

\section{Validación de un cuestionario para evaluar el uso de tecnologías para la gestión del conocimiento en estudiantes de secundaria}

\section{Validation of a questionnaire to evaluate the use of technologies for knowledge management in secondary students}

\author{
iD Dra. Sheila García-Martín \\ Profesora, Universidad de León (España) \\ iD Dra. Isabel Cantón-Mayo \\ Catedrática de Universidad, Universidad de León (España)
}

\begin{abstract}
Resumen
La gestión del conocimiento en las organizaciones educativas es un proceso que asegura el desarrollo y la aplicación de todo tipo de saberes de valor en la organización para mejorar su rendimiento y resultados. Lo cual implica, entre otros aspectos, la existencia de una infraestructura tecnológica adecuada y, la integración efectiva de las tecnologías en el proceso educativo. El objetivo del presente estudio es diseñar y validar el cuestionario Herramientas para la Gestión del Conocimiento (HEGECO) para evaluar el uso de tecnologías por parte de los estudiantes de Educación Secundaria Obligatoria y para determinar la influencia de dicho uso en sus resultados académicos. Para ello se diseña y aplica el instrumento a una muestra de 1.488 estudiantes, se calcula la validez, la fiabilidad y se realiza un análisis factorial exploratorio. Los resultados muestran que el cuestionario tiene unos niveles aceptables de fiabilidad y validez. La fiabilidad total del instrumento, estimada con el Alfa de Cronbach es de 0.80. En cuanto a la validez de constructo, se alcanza un modelo de cinco factores que explica el $75,867 \%$ de la varianza total. De manera que el presente instrumento ofrece suficientes garantías para evaluar el uso de tecnologías y sus efectos en el rendimiento académico de los estudiantes. Finalmente, se discuten sus implicaciones en la evaluación de la integración de las tecnologías en el proceso educativo y sus efectos en los resultados escolares.
\end{abstract}

\begin{abstract}
La gestión del conocimiento en las organizaciones educativas es un proceso que asegura el desarrollo y la aplicación de todo tipo de saberes de valor en la organización para mejorar su rendimiento y resultados. Lo cual implica, entre otros aspectos, la existencia de una infraestructura tecnológica adecuada y, la integración efectiva de las tecnologías en el proceso educativo. El objetivo del presente estudio es diseñar y validar el cuestionario Herramientas para la Gestión del Conocimiento (HEGECO) para evaluar el uso de tecnologías por parte de los estudiantes de Educación Secundaria Obligatoria y para determinar la influencia de dicho uso en sus resultados académicos. Para ello se diseña y aplica el instrumento a una muestra de 1.488 estudiantes, se calcula la validez, la fiabilidad y se realiza un análisis factorial exploratorio. Los resultados muestran que el cuestionario tiene unos niveles aceptables de fiabilidad y validez. La fiabilidad total del instrumento, estimada con el Alfa de Cronbach es de 0.80. En cuanto a la validez de constructo, se alcanza un modelo de cinco factores que explica el $75,867 \%$ de la varianza total. De manera que el presente instrumento ofrece suficientes garantías para evaluar el uso de tecnologías y sus efectos en el rendimiento académico de los estudiantes. Finalmente, se discuten sus implicaciones en la evaluación de la integración de las tecnologías en el proceso educativo y sus efectos en los resultados escolares.
\end{abstract}

\section{Palabras clave / Keywords}

Gestión del conocimiento; Tecnología educativa; Educación Secundaria Obligatoria; Rendimiento escolar; Evaluación; Cuestionario; Fiabilidad del test; Validez del test.

Knowledge Management; Educational technology; Compulsory Secondary Education; Academic achievement;

Evaluation; Questionnaires; Test reliability; Test validity.. 


\section{Introducción}

Las escuelas han sido definidas como organizaciones que aprenden (Bolívar 2000) y se han configurado como contextos de gestión, de realización personal y de promoción del cambio social, que centran su esencia en crear y difundir el conocimiento entre todos sus miembros (García-Martín \& Cantón, 2016).

En las organizaciones educativas, la gestión del conocimiento es un proceso que, de forma continua, asegura el desarrollo, la retención y la aplicación de todo tipo de conocimientos de valor en la organización, para mejorar su rendimiento y sus resultados. En este sentido, las investigaciones desarrolladas para estudiar la relación entre la gestión del conocimiento y el desempeño de la organización, han concluido que existe una correlación positiva y significativa entre estas dos variables (Lee \& Choi, 2003; Migdadi, 2005; Lee \& Sukoco, 2007).

A este respecto, Lee \& Choi (2003) afirmaron que los procesos de gestión del conocimiento aumentan la creatividad y el rendimiento de la organización. Otros como Cantón y Ferrero (2014) añaden el nivel de satisfacción de los usuarios. Por su parte, Migdadi (2005) y, Lee \& Sukoco (2007) concluyeron que las capacidades de gestión del conocimiento, incluida la cultura, la estructura, las personas y la tecnología influyen directamente en los procesos de gestión del conocimiento y éstos a su vez, en el rendimiento de la organización (Matin \& Sabagh, 2015).

No obstante, para la gestión efectiva del conocimiento se requiere de una combinación de factores o elementos diversos. Para Donoghue, Harris \& Weitzman (1999; citado en Taghizadeh \& Shokri, 2015) estos son: la tecnología, los recursos humanos, la cultura organizacional y la estructura organizativa. Por su parte, Stanosky \& Baldanza (2001) incorporan, además, el liderazgo y, la educación y el aprendizaje como la infraestructura básica de los procesos de gestión de conocimiento.

Al mismo tiempo, Abdullah, Selamat, Jaafar, Abdullah \& Sura (2008), quienes estudiaron la implementación de sistemas de gestión del conocimiento en instituciones de educación superior y en universidades de Malasia, incorporan la comprensión de aplicaciones y de tecnologías como elemento fundamental durante la implementación de sistemas de gestión del conocimiento.

\subsection{Las tecnologías para la gestión del conocimiento}

Para una adecuada gestión del conocimiento en las organizaciones, las tecnologías se han revelado como uno de los elementos clave. En el caso de las organizaciones educativas, el uso de las tecnologías ha ido incrementándose exponencialmente en los últimos años, llegando a considerar estas herramientas como un recurso educativo sin precedentes (Pantoja \& Huertas, 2010; citado en García-Martín \& Cantón-Mayo, 2019). Las tecnologías para la gestión del conocimiento incluyen todos aquellos medios tecnológicos que permiten a las organizaciones crear, mantener, compartir y utilizar el conocimiento de que disponen. Dichas tecnologías se centran, especialmente, en el conocimiento explícito (Rodríguez-Gómez, 2015) y requieren de una infraestructura tecnológica y de la adquisición de competencias en Tecnologías de la Información y la Comunicación (en adelante, TIC).

Las herramientas de la web 2.0 y especialmente, las herramientas colaborativas, tales como las wikis, los blogs y los chats, entre otras (Yuen \& Yuen, 2010; Zatarain-Cabada et al., 2009), en las que los usuarios además de ser consumidores, se convierten en productores de contenido son uno de los instrumentos operativos de la gestión del conocimiento (Pineda, 2014), puesto que favorecen la creación, la transferencia, el almacenamiento y la protección de los recursos de conocimiento dentro de la propia organización (Matin, \& Sabagh, 2015). De forma que, las personas y las organizaciones que los implementan deben conocer su utilidad y comprender el significado de la colaboración como proceso fundamental para la gestión del conocimiento (Flores, 2010).

El uso y la aplicación de estas herramientas en las instituciones educativas, se ha demostrado muy versátil, como muestran Bernal \& Trespaderne (2015) con el estudio de la integración de las wikis en las aulas de Educación Secundaria, así como la investigación-acción de Palazón (2013) sobre la efectividad del podcasting en la interpretación musical en alumnos adolescentes, o la de Trujillo (2011) con la introducción del podcast como herramienta educativa en un aula universitaria para favorecer el trabajo colaborativo, la capacidad crítica y la mejora de las relaciones sociales entre los estudiantes.

Sin embargo, la utilización de las herramientas para la gestión del conocimiento requiere de un conjunto de condiciones que garanticen su éxito. Puesto que, aunque la existencia de una infraestructura tecnológica adecuada es una necesidad para la gestión del conocimiento; las investigaciones que han estudiado el vínculo entre las TIC y los indicadores de desempeño en la organización no han sido concluyentes. No pudiendo 
establecer una relación directa entre las tecnologías y el desempeño de los miembros dentro de la propia organización (Emadzadeh, Mashayekhi \& Abdar, 2012; Mills \& Smith, 2010) pero sí se han obtenido implicaciones de relevancia como consecuencia de este uso en diversas variables educativas.

\subsection{Efectos del uso de tecnologías en variables educativas}

El uso cotidiano de las tecnologías por parte de los jóvenes (Austin \& Totaro, 2011; Junco, 2015; GarcíaMartín \& García-Sánchez, 2015) y la capacidad de aquellas para la conexión social de sus usuarios, ha derivado en cambios profundos en la vida de los estudiantes, ya que les permiten interaccionar entre sí para intercambiar experiencias, problemas, recursos, y, en definitiva, conocimiento (García-Peñalvo, 2016).

Por su parte, la utilización de las tecnologías para la gestión del conocimiento en las organizaciones educativas ha enriquecido los procesos de enseñanza y de aprendizaje, produciendo efectos en diversas variables educativas. La investigación de Head, Van Hoeck \& Hostetler (2017) demostró que los blogs son una fuente de aprendizaje informal para los estudiantes universitarios, puesto que les brindan información, orientación y consejos prácticos para desarrollar habilidades esenciales para la vida.

Además de incidir en el aprendizaje informal, los estudios de Bernal \& Trespaderne (2015) y de Ferrer Torregrosa, et al. (2016) indicaron que el uso de herramientas tecnológicas favorece el trabajo autónomo de los estudiantes, que aprenden a auto-gestionar su propio aprendizaje (San Martín, Peirats \& López, 2015) y que el docente pasa a desempeñar la figura de guía o de orientador durante el proceso educativo.

Otro aspecto de incidencia de las TIC en la gestión del conocimiento lo muestran investigaciones previas que señalan que la motivación hacia el aprendizaje (De Benito, Lizana \& Salinas, 2017) y la satisfacción de los estudiantes (Sánchez \& Espada, 2018) aumentaba cuando se utilizaban las tecnologías en las aulas. Así como, el estudio de Palazón (2013) destacó que variables como la satisfacción, la participación en el proceso de enseñanza y aprendizaje, y el rendimiento académico de los estudiantes se ven afectados de manera positiva por el uso de las tecnologías en las aulas. Lo cual, se confirma en los estudios internacionales de Noshahr, Talebi \& Mojallal (2014) y Wentworth \& Middleton (2014), en los que se recoge el impacto del uso de las tecnologías en el rendimiento académico de los jóvenes.

En definitiva, las tecnologías se constituyen como un recurso útil al alcance de las organizaciones educativas y además, pueden considerarse una respuesta válida a la sociedad del conocimiento en la medida en que favorecen aprendizajes continuos para todos sus integrantes y persiguen una constante transformación de la organización (Sánchez, Vial \& González 2016). Lo cual implica, entre otros aspectos, la existencia de una infraestructura tecnológica adecuada en la organización, la integración efectiva de las tecnologías en el proceso educativo y la elaboración de instrumentos que evalúen esta integración tecnológica como elementos clave en la adecuada gestión del conocimiento.

En este sentido, tras la revisión de investigaciones previas sobre la validación de escalas e instrumentos (Longás, Carrillo, Fornieles \& Riera, 2018; Múñoz-Sánchez, Azabache-Alvarado \& Quiroz, 2018; Tourón, Martín, Navarro, Pradas \& Iñigo, 2018), el propósito de este estudio fue elaborar y validar un instrumento de recogida de datos que permita evaluar el uso de tecnologías por parte de estudiantes de secundaria españoles y conocer las implicaciones de dicho uso en su rendimiento escolar. De manera que puedan establecerse conexiones positivas o no entre el uso de tecnologías y los resultados escolares.

\section{Método}

Este estudio parte de un diseño de investigación de corte cuantitativo, apoyado en un enfoque descriptivo y de correlación, en el que el método de encuesta resulta adecuado para recoger información de una amplia población y para conseguir la representatividad estadística (Bisquerra, 2014; McMillan \& Schumacher, 2010).

\subsection{Objetivo}

El objetivo de este trabajo fue diseñar y validar el cuestionario Herramientas para la Gestión del Conocimiento (HEGECO). Este instrumento fue elaborado ad hoc para evaluar el uso de herramientas tecnológicas para la gestión del conocimiento por parte de los alumnos de Educación Secundaria Obligatoria, y para determinar la influencia de dicho uso en los resultados académicos de los estudiantes en cuatro asignaturas de la etapa. 


\subsection{Población y muestra}

La población objeto de estudio estaba conformada por todos los alumnos de Educación Secundaria Obligatoria matriculados en centros educativos de las provincias de León y de Zamora en la Comunidad Autónoma de Castilla y León, durante el curso académico 2016/17.

En el curso académico anterior, 2015/16, dicha población era de 14.942 alumnos en la provincia de León y de 5.625 alumnos en la provincia de Zamora. Por tanto, un total de 20.567 alumnos conformaban la población de la presente investigación.

Para la selección de los participantes y para trabajar con un índice de confianza de $\pm 95 \%$, se calculó el tamaño muestral. Para asegurar que la muestra fuera representativa debía estar conformada por un mínimo de 375 alumnos de la provincia de León y 360 , de Zamora. De manera que, la muestra, que fue seleccionada mediante un muestreo intencional o de conveniencia quedó constituida por 1.488 alumnos de Educación Secundaria Obligatoria, de nueve centros educativos.

\section{Tabla 1}

Característica de la muestra

\begin{tabular}{|c|c|c|c|c|c|}
\hline $\begin{array}{l}\text { Variables } \\
\text { TOTAL }\end{array}$ & $\begin{array}{l}\text { № } \\
1488\end{array}$ & $\begin{array}{l}\% \\
100\end{array}$ & $\begin{array}{l}\text { Variables } \\
\text { TOTAL }\end{array}$ & $\begin{array}{l}\text { № } \\
1488\end{array}$ & $\begin{array}{l}\% \\
100\end{array}$ \\
\hline Edad & & & Curso & & \\
\hline 12 & 316 & 21.2 & $1^{\circ}$ E.S.O. & 397 & 26.7 \\
\hline 13 & 385 & 25.9 & $2^{\circ}$ E.S.O. & 403 & 27.1 \\
\hline 14 & 307 & 20.6 & 3ํㅡ.S.O. & 324 & 21.8 \\
\hline 15 & 357 & 24 & $4^{\circ}$ E.S.O. & 364 & 24.5 \\
\hline 16 & 89 & 6 & & & \\
\hline 17 & 32 & 2.2 & Centro educativo & & \\
\hline \multirow[t]{3}{*}{18} & 2 & 0.1 & Público & 617 & 41.5 \\
\hline & & & Concertado & 795 & 53.4 \\
\hline & & & Privado & 76 & 5.1 \\
\hline Sexo & & & Provincia & & \\
\hline Hombre & 698 & 46.9 & León & 1015 & 68.2 \\
\hline Mujer & 790 & 53.1 & Zamora & 473 & 31.8 \\
\hline
\end{tabular}

Fuente: Elaboración propia.

De la totalidad de la muestra, 1.015 alumnos cursaban la etapa secundaria en centros educativos ubicados en la provincia de León $(68,2 \%)$ y 473 alumnos en la provincia de Zamora $(31,8 \%)$. Asimismo, el $46,9 \%$ eran hombres y el $53,1 \%$, mujeres, de entre doce y dieciocho años. Estando distribuidos uniformemente entre los cuatro cursos de la Educación Secundaria Obligatoria, $1^{\circ}$ curso $N=397 ; 2^{\circ}$ curso $N=403 ; 3^{\circ}$ curso $N=324$; $4^{\circ}$ curso $N=364$. En la tabla 1 se muestran las principales variables demográficas de la muestra.

\subsection{Instrumento}

Una vez revisada la literatura previa contenida en la Web of Science sobre tecnologías para la gestión del conocimiento (Cabero, 2016; Rodríguez-Gómez, 2015; Rodríguez-Gómez \& Gairín, 2015) y sobre el uso de estas por parte de los jóvenes (Han \& Shin, 2016; Junco \& Cotten, 2011), se construyó el cuestionario de Herramientas para la Gestión del Conocimiento (HEGECO).

El instrumento inicial estaba configurado por 65 ítems. De los cuales, cinco ítems recogían datos sociodemográficos de la muestra: edad, curso, sexo, tipo de centro educativo y provincia, tal y como aparecen en la tabla 1. Posteriormente, siguiendo la clasificación de tecnologías para la gestión del conocimiento propuesta por Rodríguez-Gómez (2015), se incluyeron treinta ítems que hacían referencia al uso de cinco tecnologías: los motores de búsqueda, las wikis, los blogs, los podcasts y la mensajería instantánea. Finalmente, otros treinta ítems recogían información sobre el rendimiento académico de los estudiantes en cuatro asignaturas obligatorias y comunes a todos los cursos de Educación Secundaria Obligatoria: Ciencias, Matemáticas, Lengua Castellana y Literatura, y Lengua Extranjera: Inglés.

Previamente a la aplicación del instrumento y para garantizar la validez de contenido de este, el cuestionario fue enviado a cinco expertos de tres universidades españolas. El criterio para la selección de los expertos fue que contaran con experiencia en la realización de juicios y valoraciones, y en la aplicación de TIC en las aulas y/o en la implementación de procesos de gestión del conocimiento. 
Cada experto emitió un juicio crítico sobre la pertinencia, relevancia, claridad y univocidad del ítem (a partir de una escala de estimación con valores de 1 a 4) respecto a la dimensión en la que se encuadraba. En esta misma línea, se desarrolló un estudio piloto con 397 alumnos de Educación Secundaria Obligatoria de dos centros educativos, elegidos intencionalmente por las posibilidades de acceso a los mismos, para indagar en el grado de comprensión de los ítems. Como resultado de ambos procesos, se eliminaron y se realizaron modificaciones en la redacción de algunos ítems para mejorar su claridad y comprensión, hasta quedar configurada la versión definitiva del instrumento que ahora se somete a estudio.

\subsection{Procedimiento de recogida de datos}

Los centros educativos ubicados en las provincias de León y de Zamora que ofertaban la etapa de Educación Secundaria Obligatoria fueron informados del estudio. Por medio de un correo electrónico, se hizo llegar una carta informativa en la que se explicaba el propósito de la investigación y se invitaba a participar en ella de manera voluntaria. Seguidamente, se estableció contacto telefónico y presencial con los directores de los centros y se les facilitó el cuestionario en versión impresa u online, en función de la preferencia.

La cumplimentación de los cuestionarios por parte de los estudiantes tuvo lugar en las aulas, bajo la supervisión del profesorado y de uno de los investigadores. Dicha aplicación requirió de un máximo de veinte minutos por cada grupo de estudiantes para interferir lo menos posible en su proceso educativo, y se produjo durante los meses de diciembre a febrero del curso académico 2016/17.

\subsection{Análisis de datos}

Los cuestionarios fueron enumerados y eliminados los no válidos. Seguidamente, se creó una base de datos y se realizó el volcado de los datos recogidos utilizando el programa IBM SPSS Statistics, en su versión 24. Finalmente, para llevar a cabo la validación del instrumento, se calculó la fiabilidad a través del coeficiente Alfa de Cronbach y se realizó un Análisis Factorial Exploratorio con extracción de componentes principales y rotación Varimax, mediante dicho programa estadístico.

\section{Resultados}

Para comprobar la fiabilidad del instrumento se procedió al cálculo del coeficiente Alfa de Cronbach, medida de consistencia interna, que como señalan Welch \& Comer (1998) asume que los ítems miden un mismo constructo y que están altamente correlacionados. Para el cuestionario objeto de validación, el coeficiente Alfa de Cronbach arrojó un valor de $\alpha=.800$ lo que demuestra una fiabilidad elevada.

Con la intención de valorar la estructura del instrumento en función del conjunto de ítems que lo integraban, se realizó un primer Análisis Factorial Exploratorio de sus 65 ítems. La idoneidad del análisis se evaluó previamente a través del test de Kaiser-Meyer-Olkin (KMO) y de la prueba de esfericidad de Bartlett. El resultado de la prueba de adecuación muestral KMO fue de .883, con medidas de KMO individuales de las variables todas superiores a 0.7, valores considerados altos según Kaiser (1974). Además, la prueba de esfericidad de Bartlett fue estadísticamente significativa $\times 24656=68787.86(p<.001)$, lo que indicó que los datos eran probablemente factorizables. Por lo tanto, estos resultados revelaron que la aplicación del análisis factorial era apropiada.

Seguidamente, se procedió a la selección de los principales factores mediante el método de extracción de componentes principales con el objetivo de encontrar una serie de componentes que explicasen el máximo de varianza total de las variables originales.

\section{Tabla 2}

Resultados de la extracción de componentes principales

\begin{tabular}{cccc}
\hline Componente & Total & $\begin{array}{c}\text { Autovalores iniciales } \\
\text { \% de varianza }\end{array}$ & \% acumulado \\
\hline 1 & 10.831 & 11.166 & 11.166 \\
2 & 9.051 & 9.331 & 20.497 \\
3 & 6.410 & 6.608 & 27.106 \\
4 & 4.327 & 4.461 & 31.566 \\
5 & 4.294 & 4.427 & 35.994 \\
6 & 3.358 & 3.462 & 39.456 \\
7 & 3.097 & 3.192 & 42.648 \\
8 & 2.683 & 2.766 & 45.415 \\
9 & 2.364 & 2.437 & 47.852 \\
10 & 2.230 & 2.299 & 50.151 \\
\hline
\end{tabular}




\begin{tabular}{llll}
\hline 11 & 1.925 & 1.985 & 52.136 \\
12 & 1.676 & 1.728 & 53.864 \\
13 & 1.582 & 1.631 & 55.495 \\
14 & 1.488 & 1.534 & 57.029 \\
15 & 1.421 & 1.465 & 58.494 \\
16 & 1.385 & 1.428 & 59.922 \\
17 & 1.340 & 1.382 & 61.303 \\
18 & 1.290 & 1.330 & 62.634 \\
19 & 1.266 & 1.305 & 63.938 \\
20 & 1.126 & 1.161 & 65.099 \\
21 & 1.078 & 1.112 & 66.211 \\
22 & 1.026 & 1.058 & 67.268 \\
\hline
\end{tabular}

Fuente: Elaboración propia

Siguiendo la regla de normalización de Kaiser (1974), que establece la extracción de factores principales de aquellos con valor propio mayor que uno, se obtuvieron 22 componentes que explicaban el $67.268 \%$ de la varianza total, lo que podía considerarse como un valor muy aceptable. Dichos componentes o factores, se muestran en la tabla 2.

Posteriormente, se estudió el valor de la comunalidad de los ítems, con el objetivo de considerar su adecuación final, o si fuese el caso, su eliminación. De acuerdo con Zamora, Monroy \& Chávez (2009; citado en Porto-Castro, Barrero-Fernández, Gerpe-Pérez \& Mosteiro-García, 2018) las variables con una comunalidad menor a .50 carecen de explicación suficiente y no deberían ser consideradas en la interpretación del análisis. Asumiendo este criterio, el cuestionario quedó configurado por 40 ítems. A continuación, se realizó un segundo análisis factorial excluyendo las variables o ítems con una comunalidad menor a .50 con el fin de llegar a la mejor solución posible. Para comprobar la idoneidad de aplicar el Análisis Factorial Exploratorio se empleó de nuevo el estadístico Kaiser-Meyer-Olkin (KMO) y la prueba de esfericidad de Bartlett. En este segundo análisis, el resultado de la prueba de adecuación muestral KMO arrojó un valor aún más alto (.928). De igual manera, la prueba de esfericidad de Bartlett mostró resultados adecuados: $\mathrm{x} 2780=63465.938$ ( $\mathrm{p}<.001)$.

El análisis factorial mantuvo cinco componentes con valores propios todos superiores a uno y fueron capaces de explicar el $75.867 \%$ de la variabilidad total lo que puede considerarse como un valor muy aceptable. De manera que, los cinco factores resultantes que se muestran en la tabla 3 , explicaron el $75.867 \%$ de la varianza total, lo que supuso, con respecto al primer análisis factorial, un incremento del $8.59 \%$.

Tabla 3

Resultados de la extracción de componentes principales

\begin{tabular}{llll}
\hline Componente & Autovalores iniciales & \% de varianza & \% acumulado \\
\hline 1 & Total & 23.317 & 23.317 \\
2 & 9.327 & 20.057 & 43.374 \\
3 & 8.023 & 15.683 & 59.057 \\
4 & 6.273 & 8.932 & 67.989 \\
5 & 3.573 & 7.878 & 75.867 \\
\hline
\end{tabular}

Fuente: Elaboración propia.

Además, para determinar qué ítems saturaban en cada uno de los factores, se llevó a cabo la extracción de factores iniciales y se procedió a la rotación de factores a través del método de rotación ortogonal Varimax. Las variables, agrupadas por factores, que fueron obtenidas en el análisis y su correspondiente saturación, se presentan en la tabla 4.

Así pues, el cuestionario, en su versión definitiva, quedó constituido por 40 ítems agrupados en cinco factores. Respecto al primer factor que hemos denominado publicación en línea, indicar que incluyó diez ítems que explicaban el $23.317 \%$ de la varianza. Analizando el contenido de los ítems que conformaban este factor se constata que hacían referencia a una herramienta de creación y publicación de contenido textual como son los blogs, tanto a su uso, frecuencia, tiempo, lugar, finalidad y grado de satisfacción con el uso de esta herramienta.

El segundo de los factores, denominado grabación y difusión de contenido, estaba constituido por diez ítems que explicaban el $20.057 \%$ de la varianza y que hacían referencia a los podcast, una herramienta relacionada 
con la creación de contenido multimedia. A su uso, frecuencia, tiempo, lugar, finalidad y grado de satisfacción. Por su parte, el tercer factor o creación colaborativa de contenido, que explicaba el $15.683 \%$ de la varianza, estaba compuesto por diez ítems relacionados con la utilización de wikis, con su frecuencia de uso, tiempo, lugar, finalidad y grado de satisfacción.

El cuarto factor, comunicación, incluyó cinco ítems que explicaban el $8.932 \%$ de la varianza y en él se agrupaban aquellos que hacían referencia a una herramienta para la interacción social como es la mensajería instantánea, a su uso, frecuencia, lugar y grado de satisfacción con la herramienta. Finalmente, el quinto factor, rendimiento escolar, incluyó cinco ítems que explicaban el $7.878 \%$ de la varianza y concernía al rendimiento de los estudiantes, expresado a través de las calificaciones obtenidas en diferentes materias y en el trimestre y curso anteriores.

\section{Tabla 4}

Ítems que conforman cada factor y saturación correspondiente

\begin{tabular}{|c|c|c|}
\hline Factor & Ítem & Saturación \\
\hline \multirow{10}{*}{ I } & Utilizo el blog en casa & .965 \\
\hline & Grado de satisfacción con el uso del blog & .952 \\
\hline & Utilizo el blog en otros lugares & .948 \\
\hline & Uso el blog para relacionarme con otras personas & .940 \\
\hline & Utilizo el blog para divertirme & .939 \\
\hline & Tiempo de uso del blog & .929 \\
\hline & Utilizo el blog en el centro educativo & .923 \\
\hline & Frecuencia de uso del blog & .920 \\
\hline & Utilizo el blog para hacer deberes y trabajos & .913 \\
\hline & Uso el blog & .860 \\
\hline \multirow{9}{*}{ II } & Utilizo el podcast en casa & .943 \\
\hline & Uso el podcast para divertirme & .930 \\
\hline & Uso el podcast & .913 \\
\hline & Grado de satisfacción con el uso del podcast & .902 \\
\hline & Frecuencia de uso del podcast & .825 \\
\hline & Utilizo el podcast en el centro educativo & .824 \\
\hline & Utilizo el podcast para relacionarme con otros & .787 \\
\hline & Utilizo el podcast en otros lugares & .741 \\
\hline & Tiempo de uso del podcast & .733 \\
\hline \multirow{11}{*}{ III } & Utilizo el podcast para hacer deberes y trabajos & .727 \\
\hline & Utilizo las wikis para relacionarme con otros & .933 \\
\hline & Uso las wikis para hacer deberes y trabajos & .924 \\
\hline & Utilizo las wikis en casa & .923 \\
\hline & Uso las wikis & .906 \\
\hline & Uso las wikis para divertirme & .833 \\
\hline & Utilizo las wikis en otros lugares & .830 \\
\hline & Grado de satisfacción con el uso de wikis & .806 \\
\hline & Frecuencia de uso de wikis & .734 \\
\hline & Tiempo de uso de las wikis & .730 \\
\hline & Utilizo las wikis en el centro educativo & .711 \\
\hline \multirow{5}{*}{ IV } & Utilizo la mensajería instantánea en casa & .903 \\
\hline & Uso la mensajería instantánea & .893 \\
\hline & Grado de satisfacción con el uso de mensajería i. & .870 \\
\hline & Utilizo la mensajería i. para relacionarme con otr. & .846 \\
\hline & Frecuencia de uso de la mensajería instantánea & .845 \\
\hline \multirow{5}{*}{ V } & Nota media del trimestre anterior & .889 \\
\hline & Nota media del curso anterior & .831 \\
\hline & Última nota obtenida en Inglés & .810 \\
\hline & Última nota obtenida en Lengua Castellana & .800 \\
\hline & Última nota obtenida en Ciencias Sociales & .787 \\
\hline
\end{tabular}




\section{Discusión y conclusiones}

El objetivo de este estudio fue diseñar y validar un cuestionario para evaluar el uso de tecnologías para la gestión del conocimiento por parte de estudiantes de enseñanza secundaria y para conocer las implicaciones de este uso en sus resultados académicos.

En base al objetivo planteado y tras los análisis realizados y los resultados obtenidos, cabe destacar que el Cuestionario Herramientas para la Gestión del Conocimiento (HEGECO) resulta confiable y válido. La prueba Alfa de Cronbach, así como el análisis factorial exploratorio arrojan unos resultados elevados, que evidencian la fortaleza del instrumento.

El análisis factorial exploratorio de la versión definitiva del instrumento arrojó una solución de cinco factores o dimensiones que son capaces de explicar el $75,867 \%$ de la varianza total. Los resultados muestran que el número de variables que definen cada factor no varía sustancialmente, ya que los tres primeros factores están conformados por diez ítems, y los dos últimos factores por cinco ítems cada uno.

El primer factor (publicación en línea) agrupa los ítems que se refieren al uso de los blogs, una herramienta que permite crear y publicar contenido textual en la web. El uso pedagógico del blog se ha revelado como un estímulo para aprender a encontrar, a organizar y a compartir información entre el alumnado (Santos y Fernández-Río, 2012) propiciando una mayor participación de los jóvenes en su propio aprendizaje, mediante el uso de esta herramienta considerada de fácil manejo, práctica y rápida.

El segundo factor (grabación y difusión de contenido) está conformado por los ítems que hacen referencia al podcast, una herramienta para crear y publicar contenido multimedia. Dicha herramienta ha demostrado ser un recurso óptimo para el desarrollo de contenidos pedagógicos y tecnológicos en las escuelas (Trujillo, 2011). Así como también, ha confirmado ser un recurso de gran utilidad para solventar los problemas derivados de la masificación en las aulas, ya que permite al alumnado disponer de la grabación de los contenidos y de las tareas académicas, para visualizarlas, de nuevo, fuera del centro educativo y tantas veces como precise (Palazón, 2013)

Por su parte, los ítems que se refieren a las wikis y a su uso, se agrupan en el tercer factor al que hemos denominado creación colaborativa de contenido. Las wikis deben concebirse como un espacio para poner en común algo propio con los demás y así alcanzar un logro conjunto (Bernal \& Trespaderne, 2015). La aplicación de esta herramienta en el proceso educativo a través de la edición y de la creación de artículos en wikis como Wikipedia por parte de los estudiantes, ha demostrado ejercer una influencia significativa en la percepción que los jóvenes tienen de esta herramienta en términos de fiabilidad y exactitud (Soler-Adillon, Pavlovic \& Freixa, 2018).

Mientras, el cuarto factor, está conformado por los ítems relacionados con la mensajería instantánea y su utilización por parte de los estudiantes. Es preciso destacar que la mensajería instantánea es, en la actualidad, la herramienta de comunicación por excelencia entre los jóvenes, y al mismo tiempo la que menos efectos positivos registra cuando es integrada en el proceso educativo. Pues los estudios han demostrado que el uso de la mensajería instantánea durante la realización de las tareas académicas dificulta su finalización (Cotten, 2008; Junco y Cotten, 2011).

Finalmente, el quinto factor está conformado por los ítems de rendimiento académico de los estudiantes en varias asignaturas y de su rendimiento promedio. En este sentido, diversas investigaciones aseguran que el uso de la tecnología y el tipo de actividad desarrollada contribuyen a explicar, tanto el rendimiento académico de los jóvenes como su mayor éxito académico en la etapa de educación superior cuando aquellos hacen un uso equilibrado de las tecnologías (Gil, 2012; Torres-Díaz, Duart, Gómez-Alvarado, Marín-Gutiérrez, SegarraFaggioni, 2016).

De manera que los datos obtenidos permiten sugerir que es posible la utilización de este cuestionario con fines de investigación y que está en condiciones de ser un buen instrumento para estudiar la relación entre el uso de las tecnologías y el rendimiento académico en estudiantes españoles de etapas obligatorias como la educación secundaria. Únicamente a través de estos estudios, se podrá conocer no sólo la incidencia entre ambas variables, uso de tecnologías y rendimiento académico, sino también se conseguirá ahondar bajo la perspectiva de que las tecnologías generan una evolución del proceso educativo, y por ende, una transformación del papel que desempeñan tanto el alumno como el docente en la escuela del siglo XXI.

Asimismo, como fortalezas del trabajo, hay que destacar la potencia del instrumento construido para la medida de dichas variables, su relación con los indicadores de calidad a través de la satisfacción, elemento éste poco frecuente, que avanza en medida de la calidad del uso de las tecnologías para la gestión del conocimiento, así como, reseñar también, la amplitud de la muestra del estudio. Como debilidad, hay que señalar que la 
extensión provincial podría ampliarse, aunque la constancia en otros estudios señala que no hay diferencias significativas interprovinciales (Cantón, 2004).

Finalmente, como se ha venido planteando a lo largo del presente estudio, parece oportuno subrayar la necesidad de diseñar y de validar este tipo de instrumentos que permitan conocer tanto el uso de las tecnologías por parte de los estudiantes, como el impacto de dicho uso en su rendimiento y en sus resultados académicos en las diferentes etapas educativas.

Es preciso destacar que los resultados obtenidos en esta investigación tienen implicaciones significativas en el adecuado uso de las tecnologías en las aulas, ya que es importante que los docentes conozcan qué, cuándo y para qué utilizan los jóvenes las tecnologías y cuáles de dichas herramientas, usadas en las aulas, tienen influencias positivas en el rendimiento académico de los estudiantes. Además, a nivel organizativo, las escuelas, como instituciones en constante cambio y evolución, únicamente pueden ser consideradas una respuesta válida a la sociedad del conocimiento en la medida en que favorezcan aprendizajes continuos para todos sus integrantes y persigan una constante transformación de la propia organización a través del uso e implementación de las tecnologías en el proceso de enseñanza y aprendizaje con la finalidad de proporcionar una educación de calidad a todos los estudiantes.

\section{Apoyos}

Esta investigación está financiada por Universidad de León (España). Ayuda para la realización de estudios de doctorado en el marco del programa propio de investigación para 2016-2020.

\section{Referencias}

Abdullah, R. Selamat, M. H., Jaafar, A., Abdullah, S., \& Sura, S. (2008). An empirical study of knowledge management system: Implementation in Public Higher Learning Institution. IJCSNS International Journal of Computer Science and Network Security, 8(1), 281-290. https://bit.ly/2EN174l

Austin, W., \& Totaro, M.W. (2011). High School Students' Academic Performance and Internet Usage. Journal of Economics and Economic Education Research, 12(1), 41-54.

Bernal, C. \& Trespaderne, G. (2015). Wikis en la Enseñanza Secundaria. International Journal of Educational Research and Innovation (IJERI), 3, 52-63. https://bit.ly/2H24nZv

Bisquerra, R. (2014). Metodología de la investigación educativa. La Muralla.

Bolívar, A. (2000). Los centros educativos como organizaciones que aprenden. Promesa y realidades. La Muralla.

Cabero, J. (2016). La gestión del conocimiento en las organizaciones corporativas. En J. Gairín Sallán (Coord.), Aprendizaje situado y aprendizaje conectado: implicaciones para el trabajo (pp. 61-75). Wolters Kluwer.

Cantón, I. (2004). Planes de Mejora en centros educativos. Aljibe.

Cantón, I. y Ferrero, E. (2014). La gestión del conocimiento en estudiantes de magisterio. Tendencias pedagógicas, 24, 307326.

Cotten, S. R. (2008). Students' technology use and the impacts on well-being. En R. Junco \& D. M. Timm (Eds.), Using emerging technologies to enhance student engagement. New directions for student services issue 124 (pp. 55-70). Jossey-Bass. De Benito, B., Lizana, A. \& Salinas, J. (2017). Using concept mapping for faculty development in the context of pedagogic frailty. Knowledge Management \& E-Learning, 9(3), 330-347. https://bit.ly/2NGBlPo

Emadzade, M; Mashayekhi, B. \& Abdar, E. (2012). Knowledge management capabilities and organizational performance. Interdisciplinary Journal of Contemporary Research in Business, 3(11), 781-790.

Ferrer-Torregrosa, J., Jiménez Rodríguez, M.A., Torralba-Estelles, J., Garzón-Farinós, F., Pérez-Bermejo, M. \& FernándezEhrling, N. (2016). Distance learning ECTS and flipped classroom in the anatomy learning: comparative study of the use of augmented reality, video and notes. BMC Medical Education, 16(230). https://doi.org/10.1186/s12909-016-0757-3 Flores, J.C. (2010). La gestión del conocimiento y las herramientas colaborativas: una alternativa de aplicación en instituciones de educación superior. Revista de Investigación, 71(34), 11-32. https://bit.ly/2NHYQHT García-Martín, S. \& Cantón, I. (2019). Uso de tecnologías y rendimiento académico en estudiantes adolescentes. Comunicar, 59(27), 73-81. https://doi.org/10.3916/C59-2019-07

García-Martín, S. \& Cantón, I. (2016). Revisión de experiencias sobre gestión del conocimiento en organizaciones educativas. Etic@net, 239, 178-191.https://bit.ly/2TlCqCB

García-Martín, J. \& García-Sánchez, J.N. (2015). Use of Facebook, Tuenti, Twitter and Myspace among Young Spanish people. Behaviour \& Information Technology, 34(7), 685-703. https://doi.org/10.1080/0144929X.2014.993428

García-Peñalvo, F.J. (2016). La socialización como proceso clave en la gestión del conocimiento. Education in the Knowledge Society, EKS, 17(2), 7-14. https://bit.ly/2EPg975

Gil, J. (2009). Utilización del ordenador y rendimiento académico entre los estudiantes españoles de 15 años. Revista de Educación, 357, 375-396. https://bit.ly/2UgGxfr

Han, I. \& Shin, W.S. (2016). The use of a mobile learning management system and academic achievement of online students. Computers \& Education, 102, 79-89. https://doi.org/10.1016/j.compedu.2016.07.003 
Head, A.J.,Van Hoeck, M. \& Hostetler, K. (2017). Why blogs endure: A study of recent college graduate and motivations for blog readership. First Monday, 22(10). https://bit.ly/2ISWMhC

Junco, R. (2015). Student class standing, Facebook use and academic performance. Journal of Applied Developmental Psychology, 36, 18-29. https://doi.org/10.1016/j.appdev.2014.11.001

Junco, R. \& Cotten, S.R. (2011). Perceived academic effects of instant messaging use. Computers \& Education, 56, $370-378$.

https://doi.org/10.1016/j.compedu.2010.08.020

Kaiser, H. F. (1974). An index of factorial simplicity. Psychometrika, 39, 32-36. https://bit.ly/2DoXybk

Lee, H. \& Choi, B. (2003). Knowledge management enablers, processes, and organizational performance: an integrative view and empirical examination. Journal of Management Information Systems, 20(1), 179-228. https://bit.ly/2NJi7c2

Lee, L. T. \& Sukoco, B. M. (2007). The effects of entrepreneurial orientation and knowledge management capability on organizational effectiveness in Taiwan: the moderating role of social capital. International Journal of Management, 24(3), 549573. https://bit.ly/2TgE5sT

Longás, J., Carrillo, E., Fornieles, A. \& Riera, J. (2018). Desarrollo y validación del cuestionario sobre condicionantes de éxito escolar en alumnos de secundaria. Revista Española de Pedagogía, 269, 55-82. https://doi.org/10.22550/REP76-1-2018-03 McMillan, J.H. \& Schumacher, S. (2010). Investigación educativa. Pearson Addison Wesley.

Matin, E.K. \& Sabagh, P. (2015). Effects of Knowledge Management Capabilities on Organizational Performance in Iranian Export Companies. Mediterranean Journal of Social Sciences, MCSER, 6(2), 240-250.

https://doi.org/10.5901/mjss.2015.v6n2p240

Migdadi, M. M. (2005). An integrative view and empirical examination of the relationships among knowledge management enablers, processes, and organizational performance in Australian enterprises. PhD thesis. School of Economics and Information Systems, University of Wollongong.

Mills, A. M. \& Smith, T. A. (2011). Knowledge management and organizational performance: A decomposed view. Journal of Knowledge Management, 15(1), 156-171. https://doi.org/10.1108/13673271111108756

Muñoz-Sánchez, H., Azabache-Alvarado, K. \& Quiroz, M. (2018). Validez y fiabilidad del Cuestionario de Violencia Escolar para Educación Secundaria CUVE3-ESO en adolescentes peruanos. Revista de Psicología y Educación, 13(2), 142-154.

https://doi.org/10.23923/rpye2018.01.165

Noshahr, R., Talebi, B., \& Mojallal, M. (2014). The Relationship Between Use of Cell-Phone with Academic Achievement in Female Students. Applied Mathematics in Engineering, Management and Technology, 2(2), 424-428.

Palazón, J. (2013). ¿Es efectivo el podcasting/vodcasting? Investigación-acción en el aula de música en Educación Secundaria. Píxel-Bit. Revista de Medios y Educación, 43, 51-64. https://bit.ly/2EExHSi

Pineda, J. E. (2014). Social networking sites in the classroom: Unveiling new roles for teachers and new approaches to online course design. Íkala, Revista de Lenguaje y Cultura, 19(3), 269-283. https://bit.ly/2UryWLj

Porto-Castro, A.M., Barrero-Fernández, F., Gerpe-Pérez, E.M. \& Mosteiro-García, M.J. (2018). Validación de un cuestionario para evaluar el funcionamiento de las bibliotecas escolares. RELIEVE. Revista Electrónica de Investigación y Evaluación Educativa, 24(1), 1-12. https://doi.org/10.7203/relieve.24.1.12372

Rodríguez-Gómez, D. (2015). Gestión del conocimiento y mejora de las organizaciones educativas. Arco.

Rodríguez-Gómez, D., \& Gairín, J. (2015). Innovación, aprendizaje organizativo y gestión del conocimiento en las instituciones educativas. Educación, 24(46), 73-90. https://bit.ly/2cY6lsN

San Martín, A., Peirats, J. \& López, M. (2015). Las tabletas y la gestión de los contenidos digitales en los centros escolares. Revista Iberoamericana de Educación, 67, 139-158. https://bit.ly/2UlXXr4

Sánchez, M.L. \& Espada, M. (2018). Evaluación de un programa de intervención basado en el uso de las TIC para mejorar la satisfacción del alumnado hacia la Educación Física. Revista Fuentes, 20(1), 77-86.

http://dx.doi.org/10.12795/revistafuentes.2018.v20.i1.05

Sánchez, S., Vial, S. \& González, P. (2016). Producción y gestión del conocimiento. Una mirada desde el observatorio Duoc UC. En J Gairín Sallán (Coord.), Aprendizaje situado y aprendizaje conectado: implicaciones para el trabajo (pp. 483-489). Wolters Kluwer.

Santos, L. \& Fernández-Río, J. (2012). Uso pedagógico del blog: Un proyecto de investigación-acción en la materia de Educación Física en Educación Secundaria. EDUTEC. Revista Electrónica de Tecnología Educativa, 42, 1-14. https://doi.org/10.21556/edutec.2012.42.349

Soler-Adillon, J., Pavlovic, D. \& Freixa, P. (2018). Wikipedia en la Universidad: Cambios en la percepción de valor con la creación de contenidos. Comunicar, 54(26), 39-48. https://doi.org/10.3916/C54-2018-04

Stankosky, M. \& Baldanza, C. (2001). A Systems Approach on Engineering a KM system, Unpublished Manuscript.

Torres-Díaz, J.C., Duart, J.M., Gómez-Alvarado, H.F., Marín-Gutiérrez, I. \& Segarra-Faggioni, V. (2016). Usos de internet y éxito académico en estudiantes universitarios. Comunicar, 48(24), 61-70. http://dx.doi.org/10.3916/C48-2016-06

Tourón, J., Martín, D., Navarro-Asencio, E., Pradas, S. \& Iñigo, V. (2018). Validación de constructo de un instrumento para medir la competencia digital docente de los profesores (CDD). Revista Española de Pedagogía, 269, 25-54.

https://doi.org/10.22550/REP76-1-2018-02

Trujillo, J.M. (2011). Comunicación, innovación, educación y gestión del conocimiento en torno al uso del podcast en la educación superior. Revista de Universidad y Sociedad del Conocimiento, 8(2), 61-75.

http://dx.doi.org/10.7238/rusc.v8i2.1047 
Welch, S. \& Comer, J. (1998). Quantitative methods for public administration: Techniques and applications. Universidad de Virginia: Brooks/Cole.

Wentwoth, D.K., \& Middleton, J.H. (2014). Technology Use and Academic Performance. Computers \& Education, 78, $306-311$. http://dx.doi.org/10.1016/j.compedu.2014.06.012

Yuen, S. C., \& Yuen, P. K. (2010). What teachers think about Web 2.0 technologies in education? Paper presented at the 16th Annual Sloan Consortium International Conference Online Learning. Orlando, Florida.

Zatarain-Cabada, R., Barrón-Estrada, M., Zepeda-Sanchez, L., Sandoval, G., Osorio-Velázquez, J., \& Urias-Barrientos, J. (2009). Modeling student's learning styles in web 2.0 learning systems. World Journal on Educational Technology, 1(2), 78-88. 